\title{
Ubiquitin-specific protease 22-induced autophagy is correlated with poor prognosis of pancreatic cancer
}

\author{
JIN-XIAO LIANG ${ }^{1 *}$, ZHEN NING $^{2 *}$, WEI GAO ${ }^{3}$, JUN LING $^{4}$, A-MAN WANG ${ }^{2}$, \\ HAI-FENG LUO ${ }^{2}$, YONG LIANG ${ }^{5}$, QIU YAN ${ }^{3}$ and ZHONG-YU WANG ${ }^{2}$ \\ ${ }^{1}$ Zhejiang Cancer Hospital, Hangzhou, Zhejiang 310022; ${ }^{2}$ The First Affiliated Hospital of Dalian Medical University, Dalian, \\ Liaoning 116011; ${ }^{3}$ Department of Biochemistry and Molecular Biology, Dalian Medical University, Dalian, \\ Liaoning 116011, P.R. China; ${ }^{4}$ Department of Basic Sciences, The Commonwealth Medical College, Scranton, \\ PA 18509, USA; ${ }^{5}$ Department of Clinical Medicine, Taizhou University Medical School, \\ Taizhou, Zhejiang 318000, P.R. China
}

Received June 26, 2014; Accepted September 5, 2014

DOI: 10.3892/or.2014.3508

\begin{abstract}
Ubiquitin-specific protease 22 (USP22) is a component of the transcription regulatory histone acetylation complex SAGA, which broadly regulates gene transcription and correlates with cancer progression, metastasis and prognosis. Autophagy is a cell pathway with dual functions that promotes cell survival or death. However, it is not known whether USP22 can regulate autophagy in pancreatic cancer. In the present study, we first identified that USP22 was overexpressed in a large number of pancreatic cancer patient samples, concomitant with the increased expression of LC3, a marker of autophagy. Statistical analysis revealed that the increase in USP22 and autophagy was positively correlated with poor prognosis of pancreatic cancer patients. Further investigation using a human pancreatic cancer cell (Panc-1) identified that the overexpression of USP22 increased the processing of LC3 into the active form LC3-II and the number of autophagosomes, thus leading to enhanced autophagy. Activation of ERK1/2 kinase rather than AKT1 by USP22 was found to be one of the mechanisms promoting LC3 processing. USP22-induced autophagy was also found to enhance cell proliferation and resistance to starvation and chemotherapeutic drugs in Panc-1 cells, therefore expressing an overall effect that promotes cell
\end{abstract}

Correspondence to: Dr Zhong-Yu Wang, The First Affiliated Hospital of Dalian Medical University, 222 Zhongshan Road, Dalian, Liaoning 116011, P.R. China

E-mail: wangzhongyudl@gmail.com

Dr Qiu Yan, Department of Biochemistry and Molecular Biology, Dalian Medical University, 9 West Lushun South Road, Dalian, Liaoning 116011, P.R. China

E-mail: yanqiudalian@gmail.com

${ }^{*}$ Contributed equally

Key words: autophagy, LC3, pancreatic cancer, prognosis, ubiquitin-specific protease 22 survival. Collectively, the present study demonstrated a new function of USP22 that induces autophagy, thus leading to the poor prognosis of pancreatic cancer.

\section{Introduction}

Pancreatic cancer is one of the common malignances of the digestive system with an increasing incidence rate. Due to its insidious onset, the diagnosis of pancreatic cancer is usually delayed. Owing to the low resection rate, high recurrence rate, and resistance to radiotherapy and chemotherapy (1), patients with pancreatic cancer typically have a poor prognosis. Previous studies have identified 11 death-from-cancer signatures related with tumor metastasis and treatment prognosis $(2,3)$, and ubiquitin-specific protease 22 (USP22) is one of them. As a component of the transcription regulatory histone acetylation complex SAGA, USP22 regulates gene transcription at an epigenetic level through the deubiquitination of histones, exerting broad biological functions, including cell cycle progression, embryonic development and telomere homeostasis (4-6). USP22 is expressed in numerous normal human tissues but is overexpressed in malignant tumors, such as colorectal, liver, breast, gastric, bladder and lung cancer, a showing correlation with tumor progression and metastasis (7-14). Findings of these studies have demonstrated the importance of USP22 in cancer development; however, the role of USP22 in pancreatic cancer has not been investigated.

Autophagy is a self-degradative process regulating cell defense and stress response in eukaryotic systems. It is highly regulated by multiple cell signaling pathways, such as the PI3K/AKT/mTOR and Ras/RAF1/MEK1/2/ERK1/2 pathways (15-17), to respond sensitively to cellular cues. Autophagy is a double-edged sword that can either prevent or promote cancer development depending on cellular contexts. Autophagy can induce non-apoptotic or necrotic cell death or chemotherapy-induced cell death $(18,19)$. It promotes cancer cell survival under hypoxia, nutrient depletion or growth factor deprivation $(20,21)$. Thus, inhibition of autophagy can increase the sensitivity to chemotherapy, leading to cancer 
remission (22-24). Recent studies have shown that cancer cells with active autophagy tend to survive longer, causing poor prognosis of cancer $(22,23,25)$.

Pancreatic cancer is known to have a higher level of autophagy than other epithelial cancers (26). The expression of LC3 (a key structural and regulatory protein for the formation of the autophagosome) is low or absent in normal exocrine pancreas and in low-grade pancreas intraepithelial neoplasia-1 (PanIN-1) and PanIN-2 lesions. However, this expression is elevated in high-grade PanIN-3 and pancreatic ductal adenocarcinoma (PDAC), suggesting the relevance of autophagy in pancreatic cancer progression (27). Stem-like pancreatic cancer cells also show more active autophagy than less stem-like cells (28). This evidence suggests the regulatory role of autophagy in pancreatic cancer progression that can be developed into a new biomarker or therapeutic target.

Although USP22 and autophagy have been relatively well studied, their relationship in pancreatic cancer remains to be determined. In the present study, we first identified the overexpression of USP22 and LC3 in pancreatic cancer patient tissues. Using a pancreatic cancer cell line, we also demonstrated that USP22 increased LC3 processing and induced autophagy to promote cell survival. Further analysis with a large number of patient specimens identified a strong correlation between USP22-induced autophagy and the poor prognosis of pancreatic cancer.

\section{Materials and methods}

Pancreatic cancer patient samples. Pancreatic cancer tissues were collected from 68 patients during surgery at the First Affiliated Hospital of Dalian Medical University, China between 2002 and 2006. Ten adjunct non-cancerous tissues were also collected as the controls. All the tissues were fixed by formalin and embedded in paraffin wax for histological and immunohistochemical experiments. The pancreatic cancer tissues were pancreatic ductal adenocarcinoma. The cancers were staged according to the American Joint Committee on Cancer (AJCC) standards (29). All the procedures with regard to patient recruitment, informed consent, sample collection and processing were approved by the IRB of Dalian Medical University.

Reagents. Earle's balanced salt solution (EBSS), 3-methyladenine (3-MA) and monodansylcadaverine (MDC) were purchased from Sigma. LysoTracker Red and kinase inhibitors (PD98059 and LY294002) were purchased from the Beyotime Institute of Biotechnology. 2',2'-Difluorodeoxycytidine gemcitabine (GEM) was obtained from Dalian Melone, Biotechnology Co., Ltd. USP22-specific shRNA, negative control shRNA (shNC), USP22 expression construct and the blank vector were designed and synthesized by GenePharma. USP22 antibody was purchased from Abcam, LC3 antibody from Sigma, antibodies against BECN1, SQSTM1, Bcl-2, caspase-3, AKT1 from Proteintech, and antibodies against phospho-AKT1 (Ser473), ERK1/2 and phospho-ERK1/2 from Bioworld.

Cell culture and transfection. The human pancreatic cell line (Panc-1) was purchased from the Type Culture Collection of the Chinese Academy of Sciences (Shanghai, China). Panc-1 cells were cultured in Dulbecco's modified Eagle's medium (DMEM; HyClone) supplemented with $10 \%$ fetal bovine serum, $100 \mathrm{IU} / \mathrm{ml}$ penicillin and $100 \mu \mathrm{g} / \mathrm{ml}$ streptomycin at $37^{\circ} \mathrm{C}$ with $5 \% \mathrm{CO}_{2}$. Transfection of Panc-1 cells was performed using Lipofectamine 2000 reagent (Invitrogen) according to the manufacturer's instructions.

Immunohistochemical (IHC) staining. The general procedure of IHC was performed according to the protocol described (30). The USP22 antibody was used at 1:400 dilution and LC3 antibody at 1:200 dilution. Biotin-labeled secondary antibodies were used for visualization through HRP-streptavidin with 3,3'-diaminobenzidine (DAB) substrate. The counterstaining was carried out with haematoxylin. Staining with pre-immune IgG was used as the control.

Transmission electron microscopy (TEM). Panc-1 cells were collected by trypsin digestion method and fixed with $2.5 \%$ glutaraldehyde. The cell pellets were then fixed with $1 \%$ osmic acid. After a series of dehydration, cell pellets were embedded in Embedding Medium (Sigma). Ultrathin sections (50-70 nm) were prepared using Leica EM UC6 ultramicrotome and stained with uranyl acetate and lead citrate, followed by observation on a JEM-2000EX transmission electron microscope.

LysoTracker Red and MDC staining. Cells were stained with LysoTracker Red in phosphate-buffered saline (PBS) or $0.1 \mathrm{mM}$ MDC in DMEM at $37^{\circ} \mathrm{C}$ for $30 \mathrm{~min}$ in the dark. After washing three times with PBS, the cells were examined using fluorescence microscopy.

Immunofluorescence staining. Panc-1 cells were fixed with $4 \%$ paraformaldehyde, and then permeabilized with $0.1 \%$ Triton X. After blocking with goat serum, the cells were incubated with USP22 or LC3 antibody overnight at $4^{\circ} \mathrm{C}$, followed by incubation with corresponding secondary antibody for $40 \mathrm{~min}$ at room temperature. The cells were counterstained with DAPI for observation by fluorescence microscopy.

Cell proliferation assay. Cell proliferation was measured by the CCK-8 Kit (Beyotime Institute of Biotechnology) according to the manufacturer's instructions. Cells in $100 \mu 1$ media were reacted with $10 \mu \mathrm{lCCK}-8$ reagent at $37^{\circ} \mathrm{C}$ for $2 \mathrm{~h}$, followed by measuring the absorbance at $450 \mathrm{~nm}$ on a microplate reader. The data were analyzed using SPSS software.

Cell cycle assay. Cells were harvested and fixed with $70 \%$ ice-cold ethanol for $24 \mathrm{~h}$, and then stained with propidium iodide (PI) for cell cycle analysis by flow cytometry. The data were analyzed using SPSS.

Apoptosis assay. Cells were harvested and stained with the Annexin V-FITC Apoptosis Detection kit (Beyotime Institute of Biotechnology). The apoptotic cells were determined by flow cytometry and the data were analyzed using SPSS.

Reactive oxygen species (ROS) assay. Cells grown in 96-well plates were incubated with $10 \mu \mathrm{M}$ DCF-DA for $20 \mathrm{~min}$ at $37^{\circ} \mathrm{C}$. The DCF fluorescence $\left(\mathrm{Ex}_{485 \mathrm{~nm}}\right.$ and $\left.\mathrm{Em}_{535 \mathrm{~nm}}\right)$ was 


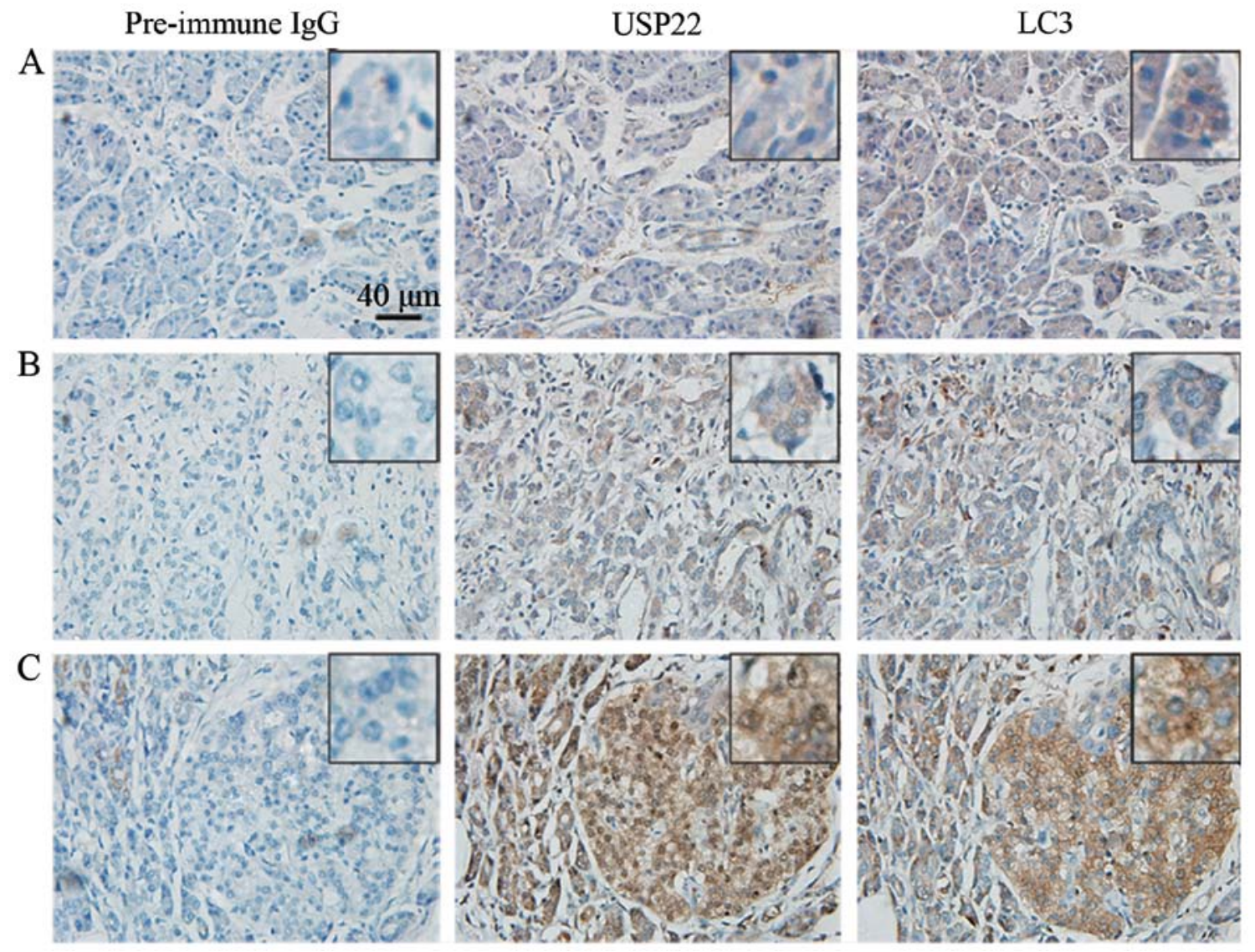

Figure 1. Detection of USP22 and LC3 expression in pancreatic cancer tissues by IHC. The experiment was performed as described in the 'Materials and methods'. Over 200 slides were performed, and the representative slides are shown. (A) Normal adjacent pancreatic tissues were used as the controls, and stained with pre-immune IgG, USP22 and LC3 antibody, respectively. (B) The pancreatic cancer tissues with a negative expression of USP22 and LC3. (C) The pancreatic cancer tissues with a positive expression of USP22 and LC3. All the images were obtained at x40 magnification with inserted panels showing the amplified images for better visualization of protein cellular localization. USP22, ubiquitin-specific protease 22; IHC, immunohistochemistry.

measured using a multimode plate reader. The data were analyzed by SPSS.

Western blotting. Cells were lysed using RIPA buffer [25 mM Tris- $\mathrm{HCl}$ (pH 7.6), $150 \mathrm{mM} \mathrm{NaCl}, 1 \% \mathrm{NP}-40,1 \%$ sodium deoxycholate, $0.1 \%$ SDS] with protease and phosphatase inhibitors (EMD Biosciences). After sonication for 2 min at $4^{\circ} \mathrm{C}$ and centrifugation for $10 \mathrm{~min}$ at $4^{\circ} \mathrm{C}$, the supernatant was taken as the total cell lysate. Protein concentration was quantified using the Bradford method. Equal amounts of total protein $(50 \mu \mathrm{g})$ were analyzed by SDS-polyacrylamide gel (SDS-PAGE). Following transfer to the NC membrane, the blot was probed with primary antibodies as indicated and then incubated with HRP-labeled secondary antibodies for visualization using enhanced chemiluminesence reagents (Thermo). The images were obtained by the Bio-Rad Imaging System.

Statistical analysis. The overall survival curves were generated using the Kaplan-Meier method. The relationship between USP 22 and LC3 in pancreatic cancer tissues was analyzed by the Spearman rank correlation analysis. The relationship between the level of LC3 or USP22 expression and clinicopathological characteristics was examined using the $\chi^{2}$ test. The differences among groups were analyzed by one-way ANOVA and the Student-Newman-Keuls (SNK)-q test using
SPSS 17.0 software. Differences were considered to indicate a statistically significant result with a P-value $<0.05$.

\section{Results}

Overexpression of USP22 correlates with a high level of autophagy in human pancreatic cancer. To examine whether USP22 is overexpressed in pancreatic cancer and determine its relationship with autophagy and pancreatic cancer prognosis, 68 pancreatic cancer tissue samples and 10 adjacent normal pancreatic tissue samples were collected and analyzed in the present study. IHC staining was used to detect USP22 and LC3 in situ. By analyzing several slides each for all 78 samples, it was found that both USP22 and LC3 were expressed in these patient samples to different extents. The representative images are shown in Fig. 1. Notably, USP22 was not expressed in normal adjacent tissues, but was overexpressed in advanced pancreatic cancer tissues, suggesting that USP22 may play an important role in pancreatic cancer progression. By contrast, LC3 was expressed at basal level in normal pancreatic tissue and also elevated to a high level in advanced pancreatic cancer tissue, indicating that it is required for physiological and pathological autophagy. In addition, it was found that USP22 was localized to the cytoplasm and nucleus, whereas LC3 was localized to the cytoplasm only, both of which are consistent with their physiological functions. 
Table I. Summary of USP22 and LC3 expression status in pancreatic cancer and adjacent normal tissues analyzed in this study.

\begin{tabular}{lcccccc}
\hline & \multicolumn{2}{c}{$\begin{array}{c}\text { USP22 } \\
\text { expression (n) }\end{array}$} & & \multicolumn{2}{c}{$\begin{array}{c}\text { LC3 } \\
\text { expression (n) }\end{array}$} & \\
\cline { 2 - 3 } Group & Neg. & Pos. & & Neg. & Pos. & Total (n) \\
\hline $\begin{array}{l}\text { Adjacent } \\
\text { normal tissue }\end{array}$ & 10 & 0 & 9 & 1 & 10 \\
$\begin{array}{l}\text { Pancreatic } \\
\text { cancer tissue }\end{array}$ & 23 & 45 & & 32 & 36 & 68 \\
\hline
\end{tabular}

Neg., negative; Pos., positive. USP22, ubiquitin-specific protease 22.

Table II. The relationship between USP22 and LC 3 expression identified by IHC experiments.

\begin{tabular}{lccc}
\hline & \multicolumn{3}{c}{ USP22 expression } \\
\cline { 2 - 4 } LC3 expression & Negative & Positive & Total \\
\hline Negative & 17 & 15 & 32 \\
Positive & 6 & 30 & 36 \\
Total & 23 & 45 & 68 \\
\hline
\end{tabular}

Spearman rank correlation analysis: $\mathrm{Q}=0.385, \mathrm{P}=0.001$. USP22, ubiquitin-specific protease 22; IHC, immunohistochemistry.

Quantitative analysis of all IHC results revealed that there were $66.2 \%$ of pancreatic cancer samples expressing USP22 and $52.9 \%$ for LC 3 . However, in adjacent normal tissues the expression of USP22 and LC 3 was 0 and $10 \%$, respectivley (Table I). The USP22 and LC3 proteins showed a significant difference between pancreatic cancer and the adjacent normal tissue $(\mathrm{P}=0.000,0.011)$. Further statistical analysis demonstrated that $44.1 \%$ of pancreatic cancer patients were USP22- and LC3-positive, whereas the percentage for double-negative in USP22 and LC3 was $25 \%$. Tumors (22.1\%) were USP22-positive but LC3-negative, while $8.8 \%$ of tumors were LC3-positive but USP22-negative (Table II). The association analysis using SPSS indicated that the correlation between USP22 and LC 3 was strong in pancreatic cancer $(\varrho=0.385$, $\mathrm{P}=0.001$ ). These results suggested that the overexpression of USP22 is highly related with autophagy in pancreatic cancer, indicating the significance of investigating the pathological role of USP22 in pancreatic cancer.

Overexpression of USP22 and the high level of autophagy correlate with the poor prognosis of pancreatic cancer patients. To clarify the relativity of USP 22 and autophagy to pancreatic cancer prognosis, we systematically analyzed the correlation of USP22 expression and autophagy with all clinicopathological characterics of pancreatic cancer from the 68 patients. The cancer stages were determined according to the AJCC system. Based on the IHC analyses of all 68 samples
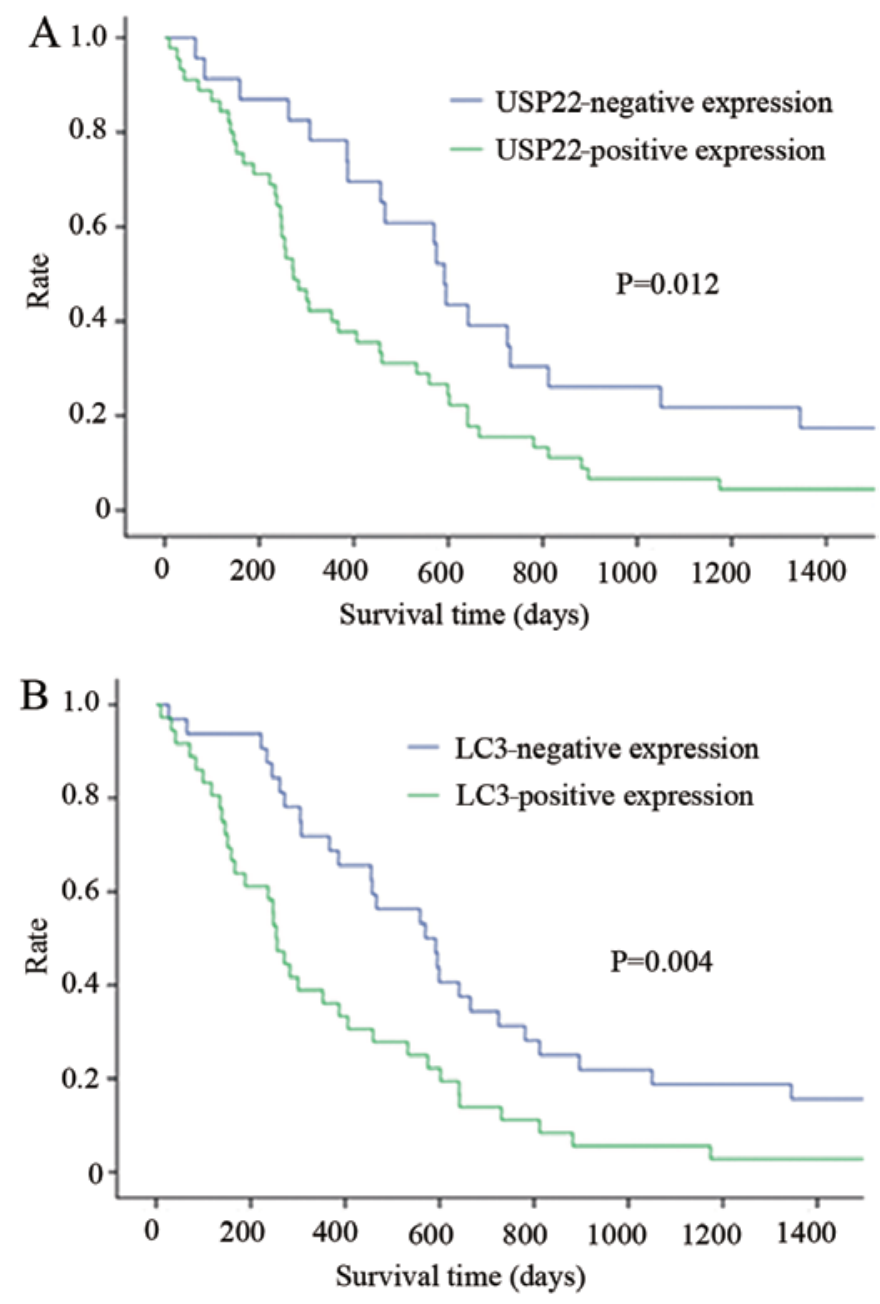

Figure 2. The survival curves of pancreatic cancer patients with various levels of USP22 and LC 3. The USP22 and LC3 protein expression data were collected from the IHC experiments in Fig. 1; the prognosis information was collected by our clinical team. The correlation was analyzed as described in the 'Materials and methods'. (A) The survival curves based on the expression level of USP22. The difference between USP22-negative and -positive groups was significant with $P=0.012$. (B) The survival curves based on the expression level of LC3. The difference between LC3-negative and -positive groups was also significant with $\mathrm{P}=0.004$. USP22, ubiquitin-specific protease 22 ; IHC, immunohistochemistry.

as above, we identified the following relationships (Table III): i) tumor differentiation, lymphatic vessel infiltration and cancer stage were associated with the expression of USP22 and LC3 $(\mathrm{P}<0.05)$; ii) pancreatic external invasion was associated with USP22 $(\mathrm{P}<0.05)$ but not with LC3 $(\mathrm{P}>0.05)$; iii) age, gender, tumor location and tumor size were not associated with the expression of USP22 and LC3 $(\mathrm{P}>0.05)$. These results proved the positive relationship between the expression of USP 22 and LC 3 and the progression of pancreatic cancer, suggesting that USP 22 overexpression may be a causal factor for pancreatic cancer.

To confirm the relationship between USP22 and LC3 and the outcome of pancreatic cancer, the survival curve of patients was analyzed using the Kaplan-Meier method. The results demonstrated that the overexpression of USP22 and LC3 was significantly correlated with short survival time (Fig. 2), indicating a close relationship between USP22 over- 
Table III. Statistical analysis of the correlation between the expression of USP22 and LC3 and the clinicopathological parameters of pancreatic cancer.

\begin{tabular}{|c|c|c|c|c|c|c|c|}
\hline \multirow{2}{*}{$\begin{array}{l}\text { Clinicopathological } \\
\text { parameters }\end{array}$} & \multirow[b]{2}{*}{$\mathrm{n}$} & \multicolumn{3}{|c|}{ USP22 expression } & \multicolumn{3}{|c|}{ LC3 expression } \\
\hline & & Neg. & Pos. & P-value & Neg. & Pos. & P-value ${ }^{a}$ \\
\hline Age (years) & & & & 0.945 & & & 0.397 \\
\hline$<60$ & 27 & 9 & 18 & & 11 & 16 & \\
\hline$\geq 60$ & 41 & 14 & 27 & & 21 & 20 & \\
\hline Gender & & & & 0.783 & & & 0.931 \\
\hline Male & 40 & 13 & 27 & & 19 & 21 & \\
\hline Female & 28 & 10 & 18 & & 13 & 15 & \\
\hline Tumor location & & & & 0.959 & & & 0.418 \\
\hline Pancreatic head & 50 & 17 & 33 & & 25 & 25 & \\
\hline Pancreatic body and tail & 18 & 6 & 12 & & 7 & 11 & \\
\hline Tumor size $(\mathrm{cm})$ & & & & 0.339 & & & 0.357 \\
\hline$<3$ & 30 & 12 & 18 & & 16 & 14 & \\
\hline$\geq 3$ & 38 & 11 & 27 & & 16 & 22 & \\
\hline Tumor differentiation & & & & 0.004 & & & 0.012 \\
\hline Well & 8 & 6 & 2 & & 7 & 1 & \\
\hline Moderate & 33 & 13 & 20 & & 17 & 16 & \\
\hline Poor & 27 & 4 & 23 & & 8 & 19 & \\
\hline Lymphatic vessel infiltration & & & & 0.007 & & & 0.019 \\
\hline Without & 41 & 19 & 22 & & 24 & 17 & \\
\hline With & 27 & 4 & 23 & & 8 & 19 & \\
\hline Pancreatic external invasion & & & & 0.02 & & & 0.096 \\
\hline Without & 31 & 15 & 16 & & 18 & 13 & \\
\hline With & 37 & 8 & 29 & & 14 & 23 & \\
\hline AJCC cancer stage & & & & 0.004 & & & 0.011 \\
\hline IA/IB/IIA & 40 & 19 & 21 & & 24 & 16 & \\
\hline IIB/III/IV & 28 & 4 & 24 & & 8 & 20 & \\
\hline
\end{tabular}

aData analysis was performed by SPSS software as described in 'Materials and methods'. Neg., negative; Pos., positive. USP22, ubiquitin-specific protease 22 .

expression and/or enhanced autophagy and the poor prognosis of pancreatic cancer patients.

USP22 induces autophagy in Panc-1 cells. Results from the clinical samples above suggested that USP22 was able to promote LC3 expression, therefore leading to autophagy. To clarify this possibility, the Panc-1 pancreatic cancer cell line was utilized for the experiments. As shown in Fig. 3A, when USP22 was downregulated by shRNA or overexpressed by USP22 cDNA construct, USP22 levels were altered accordingly. USP22 shRNA was found to markedly knock down USP22, concomitant with a notable decrease in LC3-II, indicating that USP22 was a factor for promoting LC3 processing to generate a more active form LC3-II. SQSTM1, a selective substrate of autophagy, was also decreased following USP22 overexpression, demonstrating that USP22 promoted authophagy. However, the other autophagy component beclin-1 was not affected by USP22, suggesting the early stage of autophagy may not be the target of USP22. Immunofluorescent staining experiments further supported the high efficiency of USP22 knockdown or overexpression (Fig. 3B) and the corresponding change in LC3 (Fig. 3C).

To investigate whether autophagy flux is promoted by USP22, LysoTracker Red staining (specific staining for lysosome) and MDC staining (specific staining for autophagosome) were performed. As shown in Fig. 3D, the number of lysosome and autophagosome were increased by the overexpression of USP22, suggesting that the formation of autophagosome fused with lysosome was promoted by USP22. Furthermore, TCM analysis also showed the increased number of autophagosome following the overexpression of USP22 (Fig. 3E). Taken together, these results demonstrated that USP22 is a factor that promotes autophagy in pancreatic cancer cells.

Activation of ERK is one of the mechanisms for USP22-induced autophagy. To investigate the molecular mechanism underlying the augmented autophagy by USP22, MAPK and $\mathrm{PI} 3 \mathrm{~K} / \mathrm{AKT}$ pathways were specifically analyzed in the present 


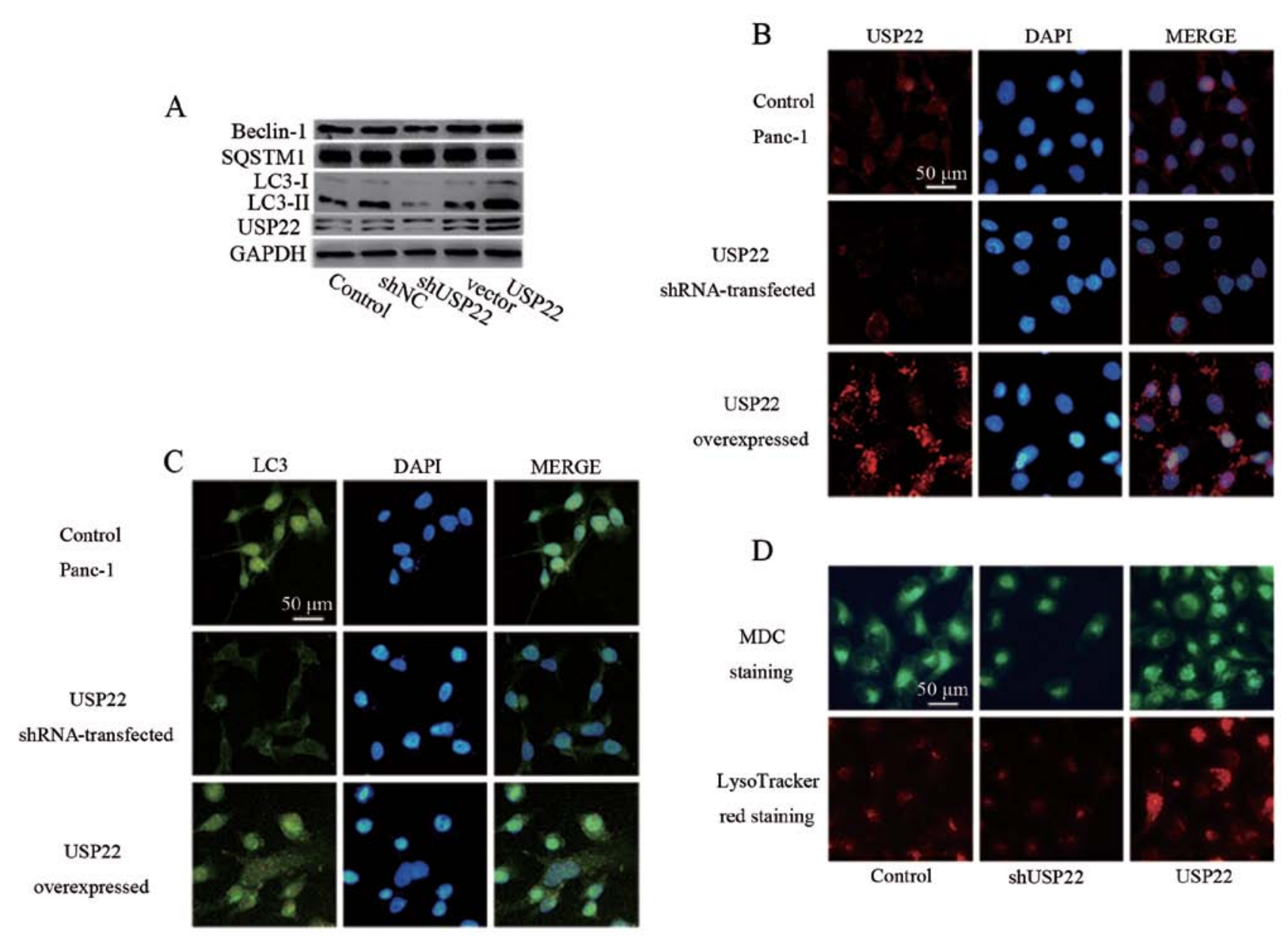

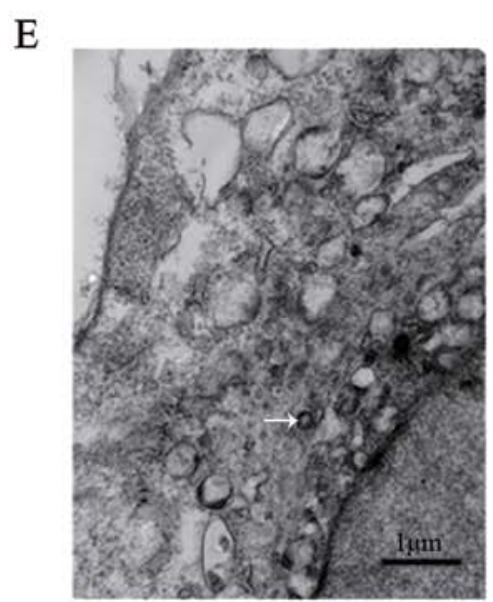

Control

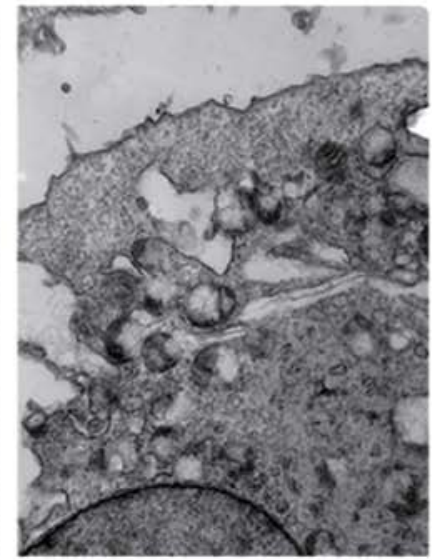

shUSP22

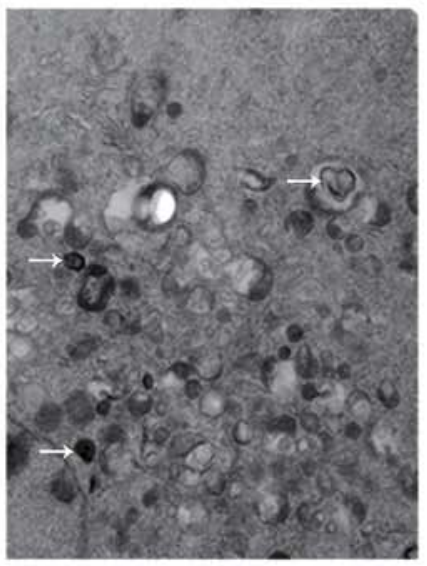

USP22

Figure 3. USP22 induces autophagy in the Panc-1 human pancreatic cancer cell. USP22 was overexpressed by cDNA expression construct and downregulated by shRNA in Panc-1 cells for the analysis of its effect on autophagy. (A) Western blot analysis with equal amounts of total cell lysate showing the expression levels of USP22 and corresponding factors involved in autophagy. (B) Immunofluorescent staining shows the amount and localization of USP22. (C) Immunofluorescent staining shows the amount and localization of LC3. (D) MDC and LysoTracker Red stainings of USP22 overexpressed and downregulated cells. (E) Analysis of autophagosome formation by TEM (x25,000). Arrows indicated autophagosomes. USP22, ubiquitin-specific protease 22; Panc-1, pancreatic cancer cell; TEM, transmission electron microscopy.

study. Using RNAi and gene overexpression combined with the application of kinase inhibitors, it was found that USP22 overexpression slightly increased ERK1/2 activity as reflected by the phosphorylated form of ERK1/2. The level of LC3-II was concomitantly increased (Fig. 4A). In addition, inactivation of ERK1/2 by a specific inhibitor (PS98059) abolished LC3-II formation, suggesting that ERK1/2 is one of the kinases involved in the promotion of LC3-II formation and subsequent autophagy, which may occur through the phosphorylation of LC3 as observed in the regulation of LC3 by PKA and PKC $(31,32)$. However, the inhibition of AKT1 by a PI3K inhibitor (LY294002) did not alter the level of LC3-II (Fig. 4B). Thus, these results demonstrated that ERK activation is involved in USP22-induced autophagy. 

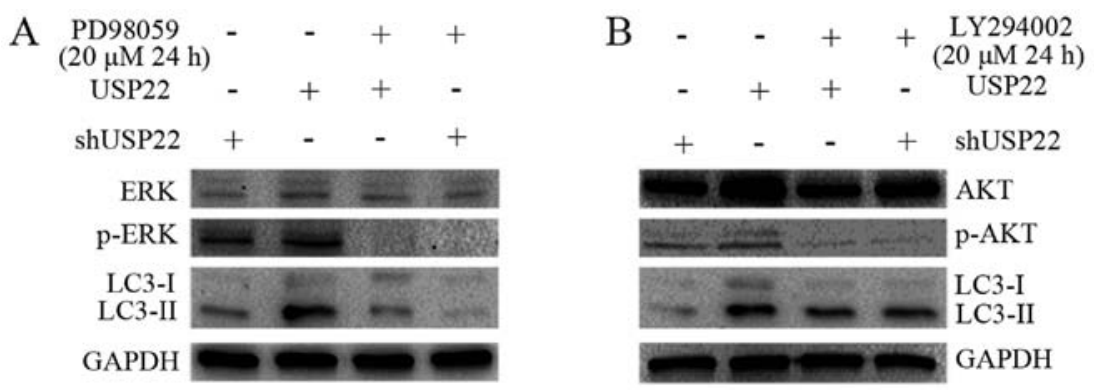

Figure 4. Involvement of ERK but not AKT1 in USP22-induced autophagy in Panc-1 cells. USP22 was upregulated or downregulated in Panc-1 cells in combination with the use of ERK inhibitor (PD98059) or PI3K inhibitor (LY294002). Equal amounts of total cell lysate were analyzed by western blotting. Western blot analysis was used to analyze the states of (A) ERK1/2 and LC3, and (B) AKT1 and LC3. USP22, ubiquitin-specific protease 22; Panc-1, pancreatic cancer cell.

USP22-induced autophagy increases Panc-1 cell survival. To elucidate the mechanism of USP22-induced autophagy on the prognosis of pancreatic cancer patients, cell death and survival pathways were further examined. When an autophagy inhibitor 3-MA was used to inhibit autophagy in Panc-1 cells, it was found to be effective as indicated by the decrease of LC3-II (Fig. 5A). The effect of USP22-induced autophagy on apoptosis was then analyzed. Western blotting of anti-apoptotic Bcl-2 and pro-apoptotic caspase-3 showed almost no difference following the overexpression or knockdown of USP22 or treatment with 3-MA (Fig. 5B). Further analysis of apoptosis with flow cytometry did not show any obvious changes (Fig. 5C, upper panel). These results demonstrated that USP22-induced autophagy has no significant effect on apoptosis in Panc-1 cells. We also examined Panc-1 cell proliferation under various levels of USP22. It was found that the proliferation of USP22 shRNA-tranfected cells was reduced by $\sim 30 \%$ as compared to USP22-overexpressed cells. The 3-MA treatment reduced the proliferation of USP22 cells by $\sim 20 \%$, but only by $10 \%$ for USP 22 shRNA-transfected cells (Fig. 5C, bottom panel). Flow cytometric analysis revealed that the percentage of S phase in USP22-overexpressed cells was increased by 2 -fold over the USP22 shRNA-transfected cells and by 1.4 -fold over the USP22-overexpressed and 3-MA-treated cells (Fig. 5D). These data confirm that the function of USP22-induced autophagy promoted Panc-1 cell proliferation.

Nutritional deficiency is a characteristic of the pancreatic cancer microenvironment (33), which is closely related to the development of pancreatic cancer $(34,35)$. To determine whether USP22-induced autophagy enhances resistance to starvation, Panc-1 cells were starved in EBSS for various timepoints. USP22-overexpressed cells showed enhanced resistance to starvation as compared to USP22 shRNA-transfected cells. However, the capacity of resistance to starvation was decreased after treatment with 3-MA (Fig. 5E), suggesting that USP22 conferred resistance to nutritional starvation is mediated by autophagy.

When Panc-1 cells were treated with a chemotherapy drug gemcitabine (36-38), it was found that the apoptotic rate of USP22-overexpressed cells was less than that of the USP22 shRNA-transfected cells. However, the apoptotic rate was increased by combinational treatment with gemcitabine and 3-MA (Fig. 5F, upper panel). The cell proliferation assay demonstrated that the inhibition rate of proliferation by USP 22 overexpression and gemcitabine treatment was less than that by the knockdown of USP22 and gemcitabine treatment. Combined treatment with gemcitabine and 3-MA further inhibited Panc-1 cell proliferation (Fig. 5F, lower panel). These results demonstrated that USP22 also promoted cell survival under chemotherapeutic condition through autophagy. Since gemcitabine functions as a nucleoside metabolic inhibitor, it generates cellular stresses, such as ROS. As shown in Fig. 5G, knockdown of USP22 plus gemcitabine treatment increased the ROS level as compared to USP22 overexpression and gemcitabine treatment. Additional treatment with 3-MA increased the level of ROS, suggesting that the prevention of ROS production by USP22 may be another mechanism for USP22-mediated cell survival.

The aforementioned results indicated that USP22-induced autophagy increases cell proliferation and the resistance to stresses, such as starvation and chemotherapy, thereby synergistically promoting the cell survival of pancreatic cancer.

\section{Discussion}

The present study aimed to identify the correlation of USP22 overexpression with poor prognosis of pancreatic cancer patients, which is mediated by the autophagy mechanism. The investigation with a large number of clinical samples ensured the medical relevance of this study, thus establishing a foundation for future clinical studies.

Systematic analyses on pancreatic cancer cell lines using various techniques have defined the central identification of this study, USP22-induced LC3 processing altering into active form LC3-II, leading to the enhanced autophagy that increased the cell survival and resistance to nutritional starvation and chemotherapy, all of which synergistically resulted in the poor prognosis of pancreatic cancer. Thus, the present study elucidates an oncogenic role rather than a tumor suppressive function of autophagy in pancreatic cancer progression. Activation of ERK1/2 was identified to be one of the mechanisms underlying the promotion of $\mathrm{LC} 3$ processing by USP22. The detailed mechanism concerning whether ERK1/2 phosphorylates LC3-I and increases its processing into LC3-II remains to be determined. However, we hypothesize that other molecular mechanisms through different signaling pathways may be involved in the regulation of USP22 effects on autophagy. Studies on AMPK and mTOR pathways may expand the association of USP22-induced 

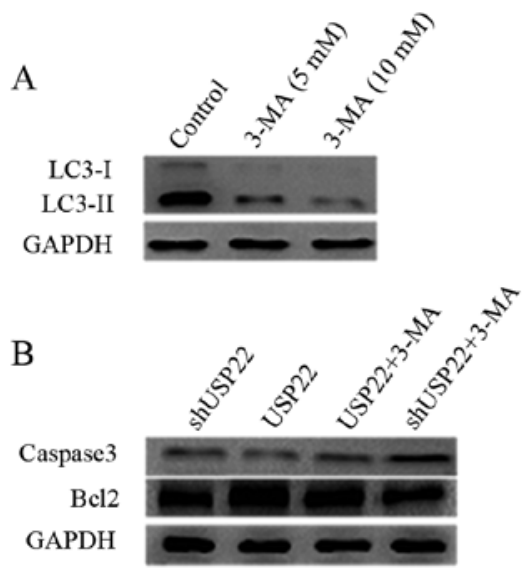

$\mathrm{D}$

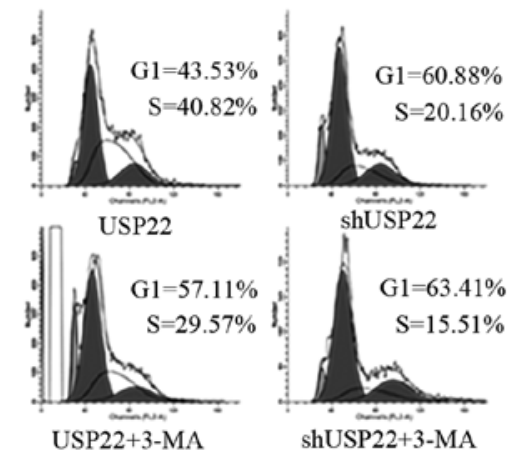

C
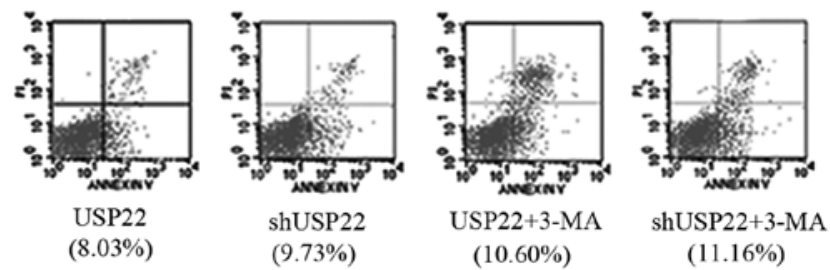

$(10.60 \%)$

$(11.16 \%)$
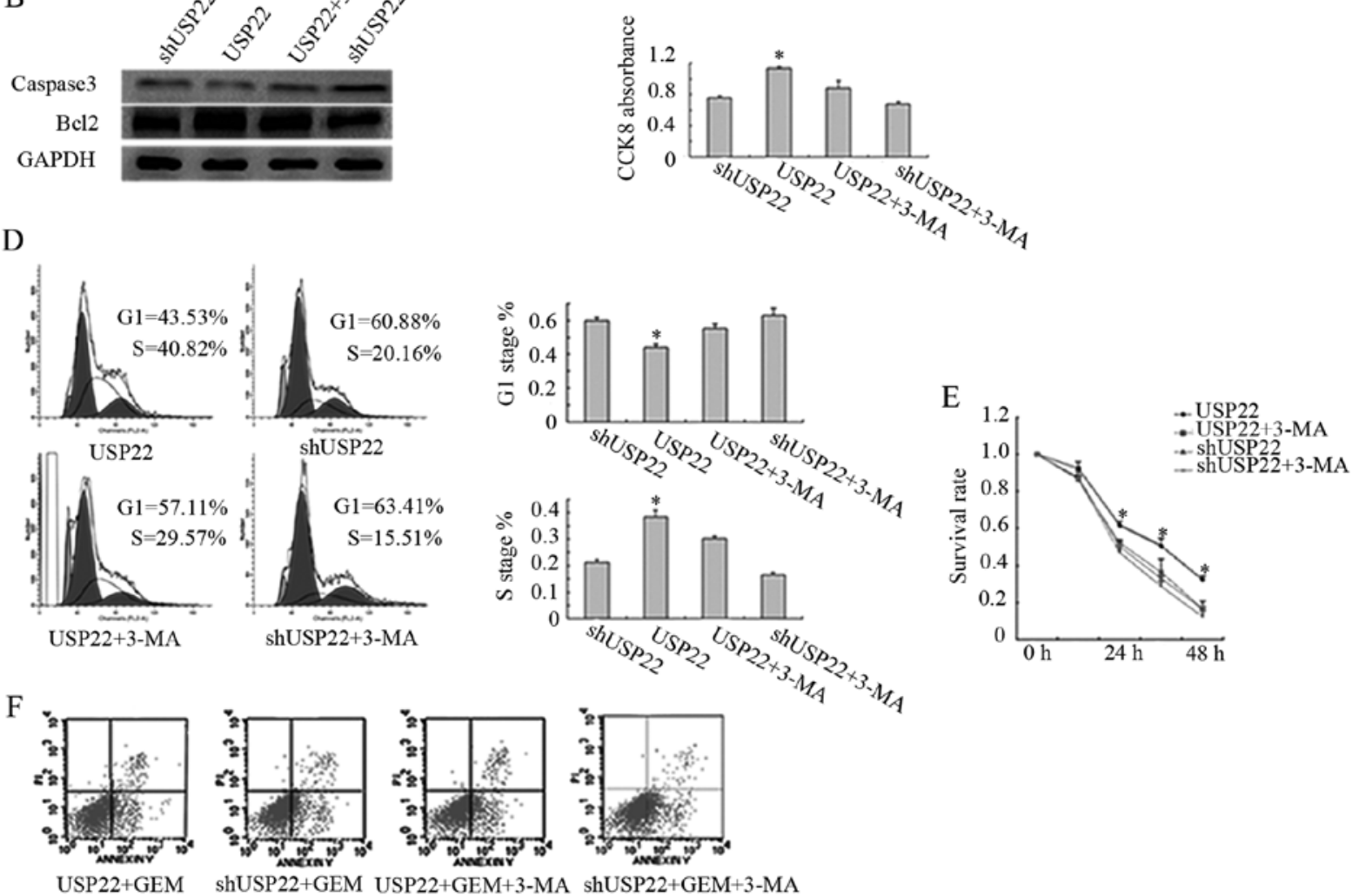

$(15.42 \%)$

$(22.37 \%)$

(20.43\%)

(23.64\%)

\section{G}
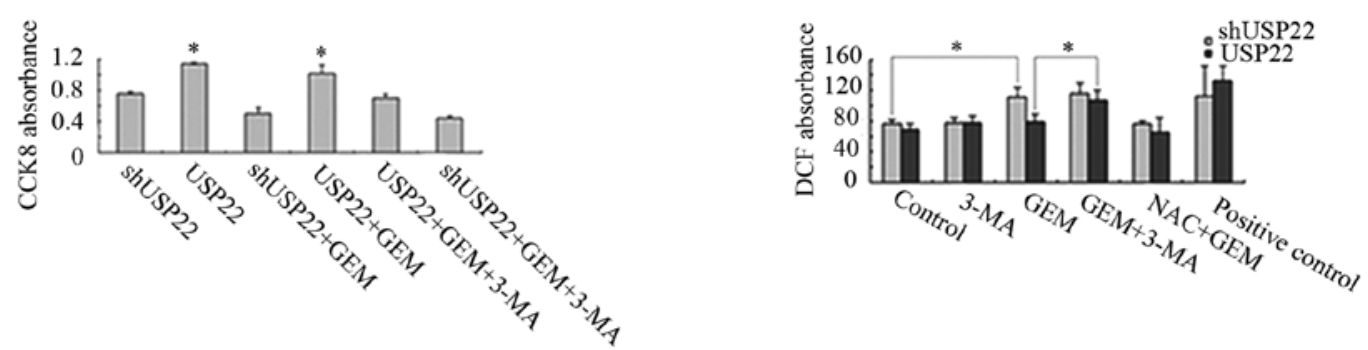

Figure 5. USP22-induced autophagy increases Panc-1 cell survival. (A) Western blotting of LC3 levels in Panc-1 cells treated with autophagy inhibitor 3-MA. (B) Analysis of caspase-3 and Bcl-2 levels in Panc-1 cells by western blotting under the upregulation or downregulation of USP22 in combination with 3-MA treatment $(10 \mathrm{mM})$. (C) Analyses of apoptosis and cell proliferation of Panc-1 cells treated by the same conditions in (B). The upper panel was the flow cytometric analysis of apoptotic cells; the bottom panel was the cell proliferation assay. (D) Flow cytometric analysis of cell proliferation based on cell cycle progression. Statistical analysis of G1 and S phases was shown by the charts to the right. (E) Measurement of cell survival rate under nutritional starvation. (F) Analyses of apoptosis and cell proliferation of Panc-1 treated with 3-MA and GEM (an antiproliferative drug for pancreatic cancer). The upper panel was the flow cytometric analysis of apoptotic cells; the bottom panel was the cell proliferation assay. (G) Measurement of ROS level under various conditions. Data presented in the charts were the mean with standard deviations. " $\mathrm{P}<0.05$ significant difference compared with ? . USP22, ubiquitin-specific protease 22 ; Panc-1, pancreatic cancer cell; 3-MA, 3-methyladenine; ROS, reactive oxygen species.

autophagy with cancer metabolomic regulation. Besides LC3, other autophagy steps or components (e.g., autophagy-related genes) may also be the targets regulated by USP22. Of note, it is still not known how USP22 regulates autophagy components at the gene transcriptional level, all of which are to be addressed in future investigations.

\section{Acknowledgements}

This study was supported by grants from the National Natural Science Foundation of China (no. 30870719), and the Dalian Municipal Science and Technology Foundation (no. 2011E15SF114). 


\section{References}

1. Gukovskaya AS and Pandol SJ: Cell death pathways in pancreatitis and pancreatic cancer. Pancreatology 4: 567-586, 2004.

2. Glinsky GV, Berezovska $\mathrm{O}$ and Glinskii AB: Microarray analysis identifies a death-from-cancer signature predicting therapy failure in patients with multiple types of cancer. J Clin Invest 115: 1503-1521, 2005.

3. Glinsky GV: 'Stemness' genomics law governs clinical behavior of human cancer: implications for decision making in disease management. J Clin Oncol 26: 2846-2853, 2008.

4. Atanassov BS, Evrard YA, Multani AS, et al: Gen5 and SAGA regulate shelterin protein turnover and telomere maintenance. Mol Cell 35: 352-364, 2009.

5. Zhang XY, Varthi M, Sykes SM, et al: The putative cancer stem cell marker USP22 is a subunit of the human SAGA complex required for activated transcription and cell-cycle progression. Mol Cell 29: 102-111, 2008.

6. Zhao Y, Lang $\mathrm{G}$ and Ito S, et al: A TFTC/STAGA module mediates histone $\mathrm{H} 2 \mathrm{~A}$ and $\mathrm{H} 2 \mathrm{~B}$ deubiquitination, coactivates nuclear receptors, and counteracts heterochromatin silencing. Mol Cell 29: 92-101, 2008.

7. Lee HJ, Kim MS, Shin JM, Park TJ, Chung HM and Baek KH The expression patterns of deubiquitinating enzymes, USP22 and Usp22. Gene Expr Patterns 6: 277-284, 2006.

8. Liu YL, Yang YM, Xu H and Dong XS: Increased expression of ubiquitin-specific protease 22 can promote cancer progression and predict therapy failure in human colorectal cancer. J Gastroenterol Hepatol 25: 1800-1805, 2010.

9. Liu YL, Yang YM, Xu H and Dong XS: Aberrant expression of USP22 is associated with liver metastasis and poor prognosis of colorectal cancer. J Surg Oncol 103: 283-289, 2011.

10. Yang DD, Cui BB, Sun LY, et al: The co-expression of USP22 and BMI-1 may promote cancer progression and predict therapy failure in gastric carcinoma. Cell Biochem Biophys 61: 703-710, 2011.

11. Zhang Y, Yao L and Zhang X, et al: Elevated expression of USP22 in correlation with poor prognosis in patients with invasive breast cancer. J Cancer Res Clin Oncol 137: 1245-1253, 2011.

12. Lv L, Xiao XY, Gu ZH, Zeng FQ, Huang LQ and Jiang GS: Silencing USP22 by asymmetric structure of interfering RNA inhibits proliferation and induces cell cycle arrest in bladder cancer cells. Mol Cell Biochem 346: 11-21, 2011.

13. Ning J, Zhang J, Liu W, Lang Y, Xue Y and Xu S: Overexpression of ubiquitin-specific protease 22 predicts poor survival in patients with early-stage non-small cell lung cancer. Eur J Histochem 56 e46, 2012.

14. Ling SB, Sun DG and Tang B, et al: Knock-down of USP22 by small interfering RNA interference inhibits HepG2 cell proliferation and induces cell cycle arrest. Cell Mol Biol 58 (Suppl): OL1803-OL1808, 2012.

15. Vivanco I and Sawyers CL: The phosphatidylinositol 3-kinase AKT pathway in human cancer. Nat Rev Cancer 2: 489-501, 2002.

16. Ogier-Denis E and Codogno P: Autophagy: a barrier or an adaptive response to cancer. Biochim Biophys Acta 1603 113-128, 2003

17. Pattingre $\mathrm{S}$, Bauvy $\mathrm{C}$ and Codogno $\mathrm{P}$ : Amino acids interfere with the ERK1/2-dependent control of macroautophagy by controlling the activation of Raf-1 in human colon cancer HT-29 cells. J Biol Chem 278: 16667-16674, 2003
18. Pardo R, Lo Ré A, Archange C, et al: Gemcitabine induces the VMP1-mediated autophagy pathway to promote apoptotic death in human pancreatic cancer cells. Pancreatology 10: 19-26, 2010.

19. Tung WL, Wang Y, Gout PW, Liu DM, Gleave M and Wang Y: Use of irinotecan for treatment of small cell carcinoma of the prostate. Prostate 71: 675-681, 2011.

20. Mathew R, Karantza-Wadsworth V and White E: Role of autophagy in cancer. Nat Rev Cancer 7: 961-967, 2007.

21. Debnath J, Baehrecke EH and Kroemer G: Does autophagy contribute to cell death? Autophagy 1: 66-74, 2005.

22. Li J, Hou N, Faried A, Tsutsumi S and Kuwano H: Inhibition of autophagy augments 5-fluorouracil chemotherapy in human colon cancer in vitro and in vivo model. Eur J Cancer 46: 1900-1909, 2010.

23. Liu F, Liu D, Yang Y and Zhao S: Effect of autophagy inhibition on chemotherapy-induced apoptosis in A549 lung cancer cells. Oncol Lett 5: 1261-1265, 2013.

24. Shingu T, Fujiwara K, Bögler O, et al: Inhibition of autophagy at a late stage enhances imatinib-induced cytotoxicity in human malignant glioma cells. Int J Cancer 124: 1060-1071, 2009.

25. Fujii S, Mitsunaga S, Yamazaki M, et al: Autophagy is activated in pancreatic cancer cells and correlates with poor patient outcome. Cancer Sci 99: 1813-1819, 2008.

26. Yang S and Kimmelman AC: A critical role for autophagy in pancreatic cancer. Autophagy 7: 912-913, 2011.

27. Yang S, Wang X, Contino G, et al: Pancreatic cancers require autophagy for tumor growth. Genes Dev 25: 717-729, 2011.

28. Rausch V, Liu L, Apel A, et al: Autophagy mediates survival of pancreatic tumour-initiating cells in a hypoxic microenvironment. J Pathol 227: 325-335, 2012.

29. Egner JR: AJCC cancer staging manual. JAMA 304: 1726-1727, 2010.

30. Gillett CE: Immunohistochemistry. Methods Mol Med 120: 191-200, 2006.

31. Cherra SR III, Kulich SM, Uechi G, et al: Regulation of the autophagy protein LC3 by phosphorylation. J Cell Biol 190: 533-539, 2010.

32. Jiang $\mathrm{H}$, Cheng $\mathrm{D}$, Liu $\mathrm{W}$, Peng $\mathrm{J}$ and Feng $\mathrm{J}$ : Protein kinase $\mathrm{C}$ inhibits autophagy and phosphorylates LC3. Biochem Biophys Res Commun 395: 471-476, 2010.

33. Levine B and Klionsky DJ: Development by self-digestion: molecular mechanisms and biological functions of autophagy. Dev Cell 6: 463-477, 2004.

34. Guerra C, Collado M, Navas C, et al: Pancreatitis-induced inflammation contributes to pancreatic cancer by inhibiting oncogene-induced senescence. Cancer Cell 19: 728-739, 2011.

35. Neesse A, Michl P, Frese KK, et al: Stromal biology and therapy in pancreatic cancer. Gut 60: 861-868, 2011

36. Burris HR, Moore MJ, Andersen J, et al: Improvements in survival and clinical benefit with gemcitabine as first-line therapy for patients with advanced pancreas cancer: a randomized trial. J Clin Oncol 15: 2403-2413, 1997.

37. Donadelli M, Dando I, Zaniboni T, et al: Gemcitabine/cannabinoid combination triggers autophagy in pancreatic cancer cells through a ROS-mediated mechanism. Cell Death Dis 2: e152, 2011.

38. DeNicola GM, Karreth FA, Humpton TJ, et al: Oncogene-induced Nrf2 transcription promotes ROS detoxification and tumorigenesis. Nature 475: 106-109, 2011. 